\title{
Analysis of Interwell Tracer Flow Behaviour Through Heterogeneous Layered Reservoirs
}

\author{
Yi T., Daltaban T. S., Archer J. S. \\ Imperial College of Science, U.K.
}

\begin{abstract}
Copyright 1995, Steering Committee of the European IOR - Symposium.
This paper was presented at the 8th. European IOR - Symposium in Vienna, Austria, May 15 - 17, 1995

This paper was selected for presentation by the Steering Committee, following review of information containad in an abstract

submitted by the author(s). The paper, as presented has not been reviewed by the Steering Committee.
\end{abstract}

\begin{abstract}
Tracer tests can be used to investigate reservoir heterogeneity which we define as reservoir characteristics which effect the sweep efficiency and fluid flow behaviour on a scale of practical interest, and which can be very important information especially for the design of IOR applications. It is helpful to understand the mechanism of tracer transport in various heterogeneous reservoirs before any interpretation of tracer test result is used to deduce reservoir heterogeneity.
\end{abstract}

In this paper, a new numerical approach is applied to model interwell tracer flow paths in heterogeneous porous media. In order to conserve mass and to reduce numerical dispersion to an insignificant degree, special techniques are described. As a result, both physical dispersion and adsorption, which can have significant effects on tracer transport in porous media, can be simulated efficiently. The techniques used include a mixed finite element method for solving the velocity and pressure fields simultaneously, a mass balance method for the solution of the phase saturation, and a random walk approach for simulating the tracer transport. Mechanisms of tracer transport in realisation of reservoirs with vertical and areal heterogeneities have been analysed using the model.

\section{Introduction}

Interwell tracer tests involve injecting a slug of tracer fluid into an injection well, and recording the tracer production profiles at the producers. When the tracer fluid flows through the reservoir towards the producers, it experiences all the characteristics of the reservoir that it encounters. Thus, the path of the tracer fluid would represent the sweep patterns for the reservoir fluids, and the tracer concentration profiles obtained from the producers should reflect the reservoir characteristics. Tracer tests have been used to investigate reservoir heterogeneity ${ }^{1,2.3}$, such as vertical layering, areal permeability variations and fractures. The tracer may partition on the aqueous or hydrocarbon phases and some advantages can be gained by using mixed 
tracers. Reservoir characteristics from tracer têsts can be very important, especially for the design of infill well locations and for IOR applications.

Any interpretation of tracer test results is enhanced by knowledge and understanding of the mechanism of tracer transport in porous media with different characteristics. The numerical modelling of tracer flow can illuminate such understanding and can then help in planning the design and interpretation of tracer tests and in defining infill well locations.

In this paper, the mixed finite element method and the random walk approach ${ }^{4,5}$ are used to simulate the interwell tracer flow through heterogeneous reservoirs. Pressure distribution, flow paths and tracer concentration profiles are predicted for heterogeneous reservoirs using our newly developed model. The heterogeneity in a vertical direction is characterised by multi-layers of different $\mathrm{kh}$ values. Different permeability patterns are used to represent the areal heterogeneity.

\section{Numerical Models}

Numerical models for the tracer flow should honour the physical characteristics of the tracer test process. In tracer tests, the volume of tracer fluid injected is normally very small compared with the volume of reservoir fluids, and physical dispersion and adsorption may have significant effects on the tracer fluid flow. Two problems that appear regularly in reservoir simulation, need to be solved in the numerical model used for simulating the tracer fluid flow. One is to reduce the numerical dispersion to a negligible degree so that the physical dispersion can be properly simulated; the other is to ensure mass conservation, otherwise the small volume of tracer could become lost, resulting in erroneous prediction of tracer response. Our new model has been developed to meet these requirements by using a mixed finite element method and a random walk model. We have previously discussed the models in Refs. (4) and (5), and will not repeat it again here except as a brief summany: We intend in this paper to concentrate on application of the code.

Our simulator was developed for modelling tracer flow in two dimensional two phase (water and oil) flow in a slightly compressible heterogeneous reservoir. The mixed finite element model solves the pressure and velocity fields simultaneously. Boundary conditions for both pressure and velocity are imposed in the process of solution, therefore the calculated pressure and velocity can satisfy the boundary conditions strictly. For instance, the magnitude of the velocity normal to a no-flow boundary is zero. The mass balance principle is then applied to solve for the phase saturation. In the random walk model, the tracer fluid is represented by a number of particles. Based on the velocity field predicted from the mixed finite element method, the random walk model transports and tracks the tracer fluid particles. It accounts for dispersion (including molecular diffusion, longitudinal and transverse dispersion) and adsorption through the reservoir and towards the production well, and records the particle production profile at the production well. From the random. walk process, one can picture the tracer movement through the reservoir and the way it responds to heterogeneities. This provides the corresponding tracer concentration profile.

\section{Results and Discussion}

We use the present models to simulate tracer transport through reservoirs in the presence of complicated heterogeneities. We analyse some relevant cases, including a quarter five-spot pattern and a line-drive pattern, both of which have different permeability distributions, and a commingled multi-layered quarter five-spot pattern.

\subsection{The quarter five-spot pattern}

As the first example, we look at the simplecase of a quarter five-spot pattern with no-flow boundaries. Tracer fluid is injected in one 


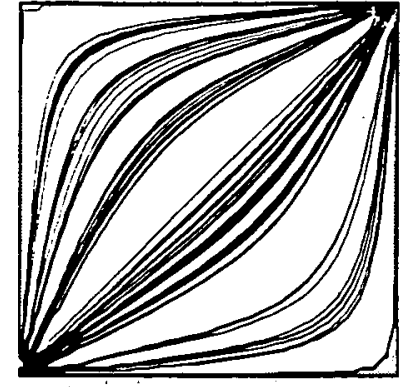

Flow paths

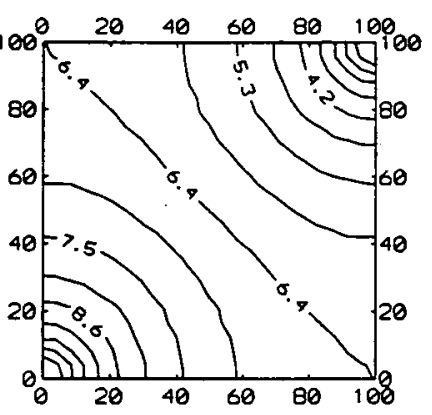

Pressure contours (a) homogeneous $(\mathrm{k}=1 \mathrm{md})$

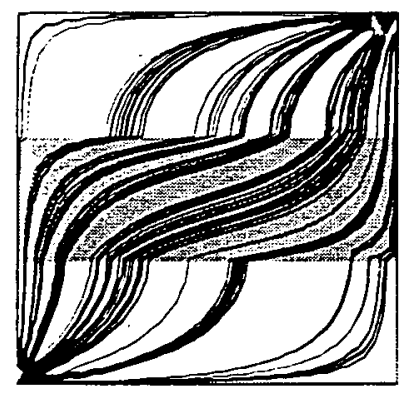

Flow paths

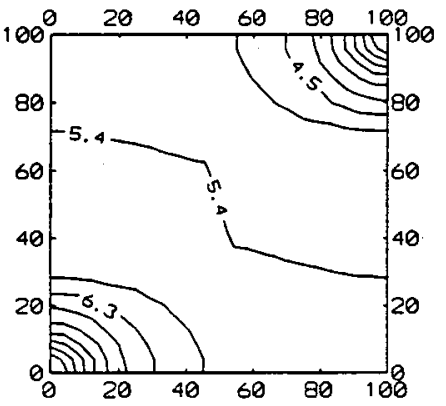

Pressure contours (b) with a higher-k (100 md) streak

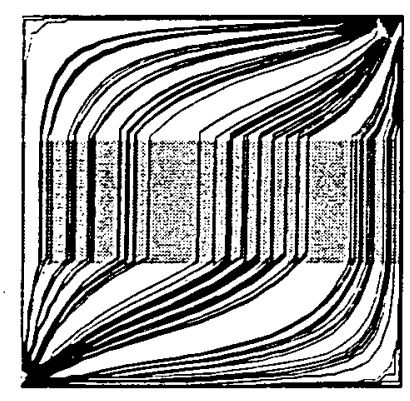

Flow paths

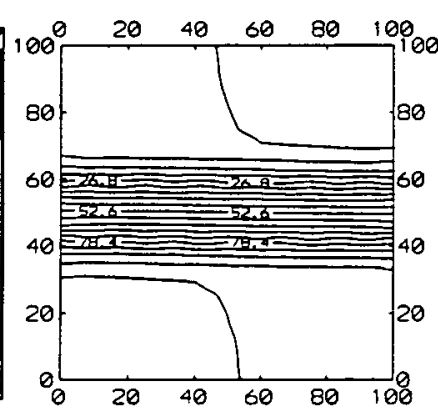

Pressure contours (c) with a lowerer-k $(0.01 \mathrm{md})$ streak

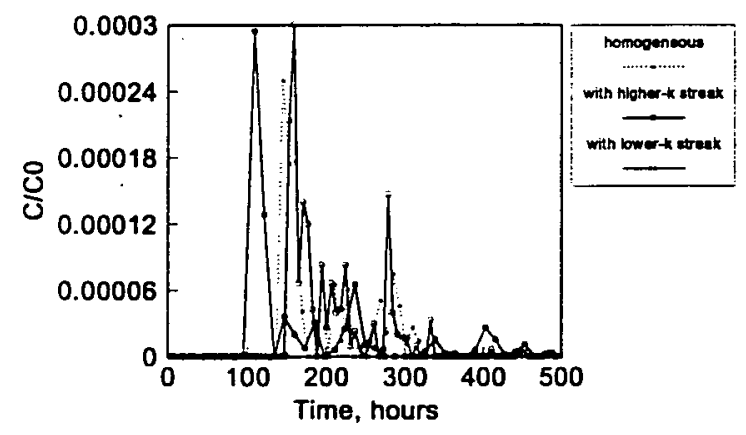

(d) Comparison of tracer concentration profiles

Figure 1 - A quarter five-spot pattern with heterogeneities corner and produced in the opposite corner. For the sake of comparison, cases with different permeability distributions are analysed. We have included a homogeneous case and cases with different permeability heterogeneity patterns. The pressure contours and tracer flow paths for the homogeneous case are shown in Figure 1a. It may be seen that the pressure contours are symmetrical about the diagonal. For our first permeability heterogeneity, we have assumed a higher permeability rectangular zone in the middle of the pattern. Figure $1 \mathrm{~b}$ shows the pressure contours and the tracer flow paths for this case. It is clear that the symmetry of pressure contours in the homogeneous case are distorted by the high permeability region in this more heterogeneous case; and the direction of tracer flow is changed by the higher permeability streak, bending it towards $\mathrm{x}$-direction. If the higher permeability streak is now replaced by a lower permeability streak, the tracer flow paths and pressure contours are illustrated in Figure 1c. There it can be seen that the tracer flows mainly in y-direction in the lower permeability region as might be expected. The tracer concentration profiles corresponding to the homogeneous case and the above two heterogeneous cases are compared in Figure 1d. It is clear that the higher permeability region induces an earlier breakthrough of tracer, but production of tracer lasts longer than in the homogeneous case. The lower permeability streak delays the tracer breakthrough. This accords with physical expectations.

Let us now consider isolated lower and higher permeability zones introduced into the pattern. The tracer flow paths and pressure contours in the reservoir and the tracer concentration responses at the production well are shown in Figures $2 \mathrm{a}$ through $2 \mathrm{c}$. We see that the higher permeability zone draws the flow towards it, while the lower permeability zone repels the tracer flow. In terms of tracer concentration response, the isolated higher permeability zone in this case reduces the tracer breakthrough time in the same way as the rectangular higher permeability streak did in the previous case. 


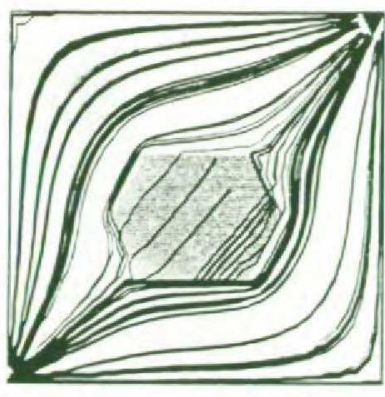

Flow paths

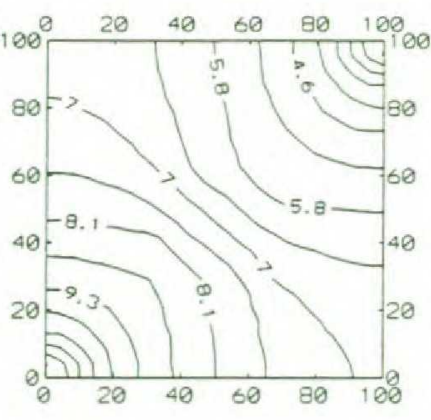

Pressure contours (a) with a lower-k ( $0.01 \mathrm{md})$ zone in the centre

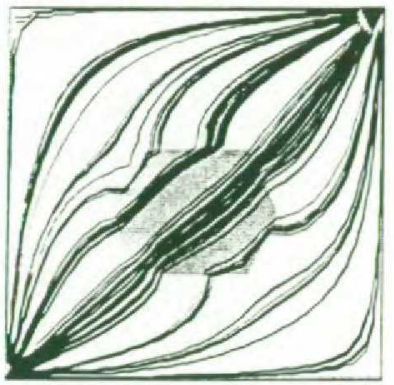

Flow paths

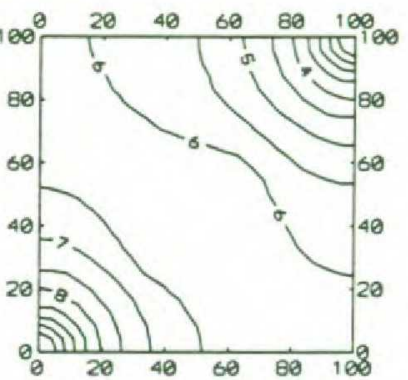

Pressure contours (b) with a higher-k (100 md) zone in the centre

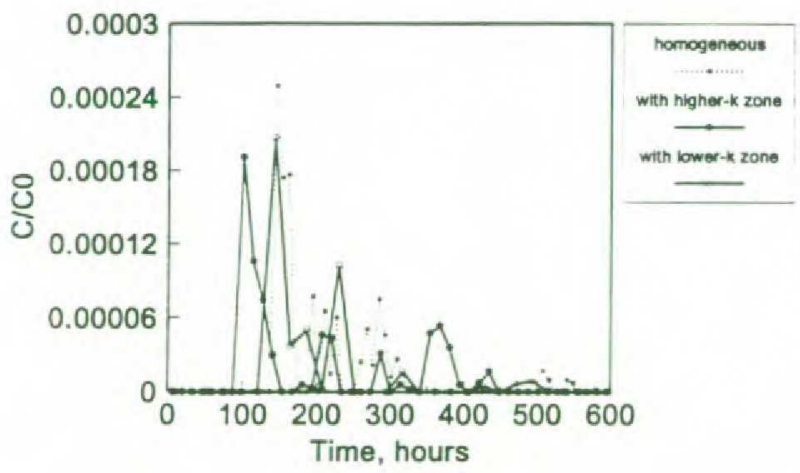

(c) Comparison of tracer concentration profiles

Figure 2 - A quarter five-spot pattern with permeability heterogeneities

The isolated lower permeability zone however had little effect on the breakthrough time but it did reduce the peak value of the concentration profile and increase the time for the production of tracer. The reason for this is that most of the tracer flows around the lower permeability zone towards the producer, but the tracer flows very slowly once it enters into the lower
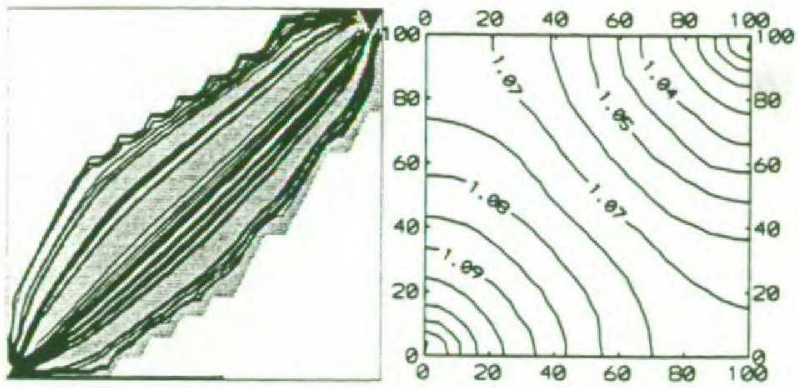

Flow paths

\section{Pressure contours}

(a) with a higher-k ( $100 \mathrm{md}$ ) zone
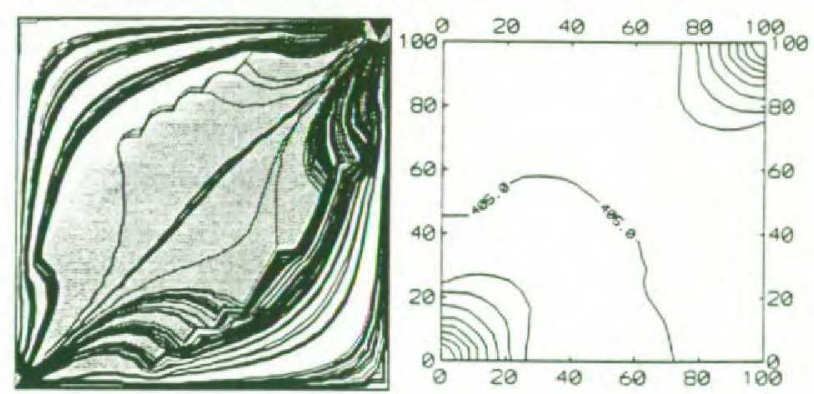

Flow paths

Pressure contours

(b) with a lower-k ( $0.01 \mathrm{md})$ zone

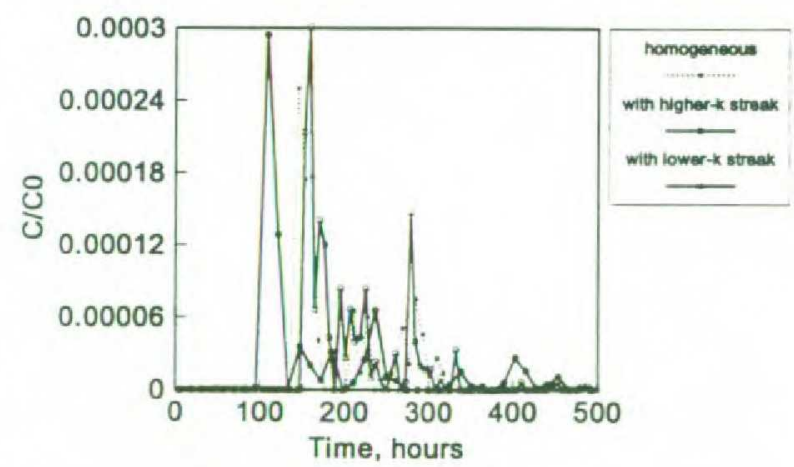

(c) Comparison of tracer concentration profiles

Figure 3 - A quarter five-spot pattern with heterogeneities

permeability zone.

If we model continuity within the heterogeneous regions through to the wells, the effect of the heterogeneity patterns on tracer flow and on tracer concentration response can be seen in Figures $3 \mathrm{a}$ through $3 \mathrm{c}$. It can be seen that in the presence of a lower 
permeability region, most of the tracer flow bypasses the lower permeability region, and then re-enters the region near the production well. In the presence of a higher permeability region, the tracer flow takes a short cut through the higher permeability region and quickly appears at the production well. We see the familiar result of an early tracer breakthrough accompanied by a higher peak value of concentration. It is interesting to recognise the influence of the lower permeability zone in diverting flow so that the tracer breakthrough is earlier than in the homogeneous case. This is reasonable once it is recognised that with a no-flow boundary condition, the lower permeability region reduces the effective cross-sectional area of the tracer flow compared with the homogeneous case.

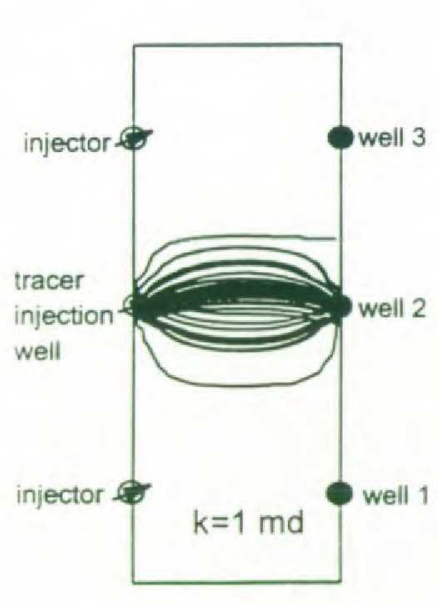

a) Tracer flow lines

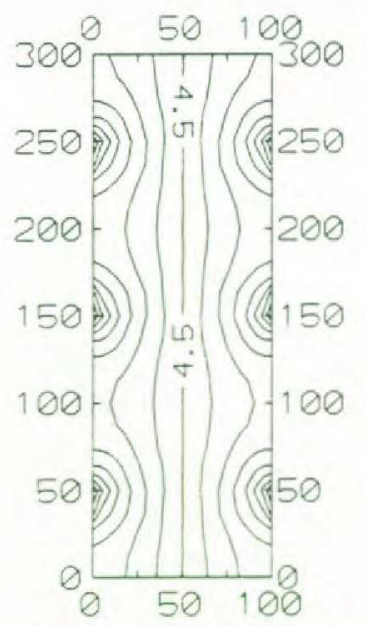

b) Pressure contours

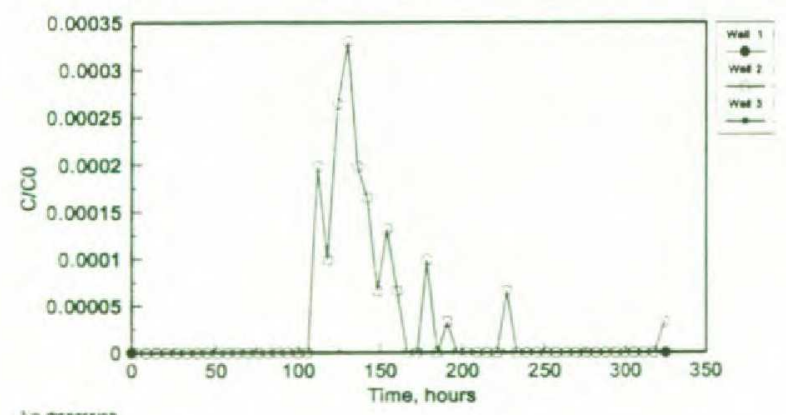

somogerson

c) Tracer concentration profiles

\subsection{A line-drive pattern}

In the cases described above only one production well is involved in the quarter of five spot pattern. We now look at how heterogeneity influences tracer flow and concentration response in a direct line drive pattern where there are three injection wells and three production wells. In that system the tracer is injected from the middle injector, and it could possibly be produced at any of the production wells. In first case for this pattern, we assumed a homogeneous permeability of 1 md. The tracer flow paths and the pressure contours predicted are shown in Figure 4a and $4 \mathrm{~b}$, and the concentration responses from the three wells are illustrated in Figure 4c. We show that the tracer flows immediately to the middle well, and there is no tracer production

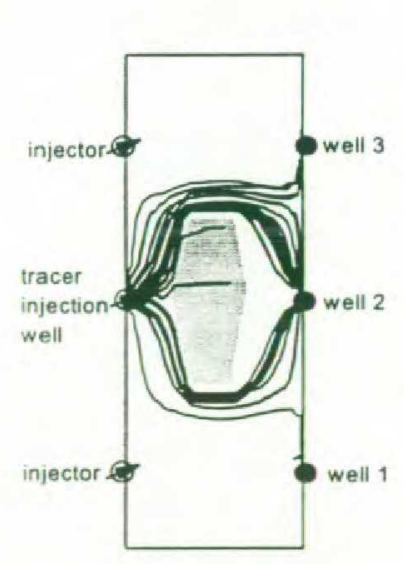

a) Tracer flow lines

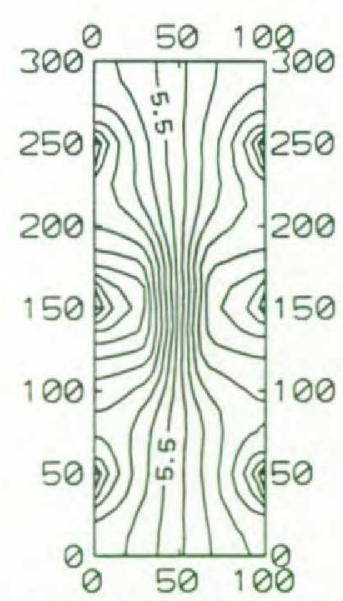

b) Pressure contours

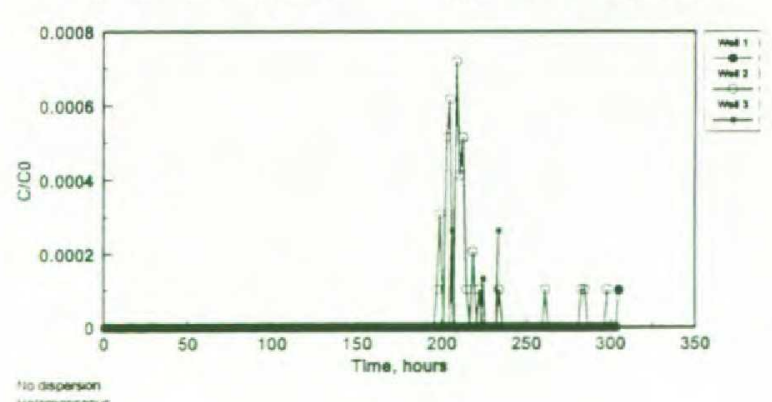

c) Tracer concentration profiles

Figure 4 - A homogeneous line drive pattern

Figure 5 - A line drive pattern with a lower-k $(0.01 \mathrm{md})$ zone in the centre 

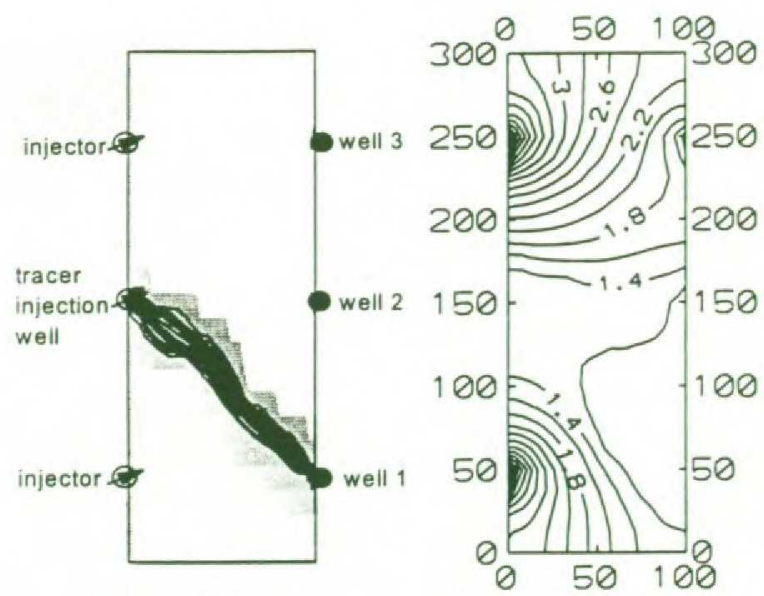

a) Tracer flow lines

b) Pressure contours

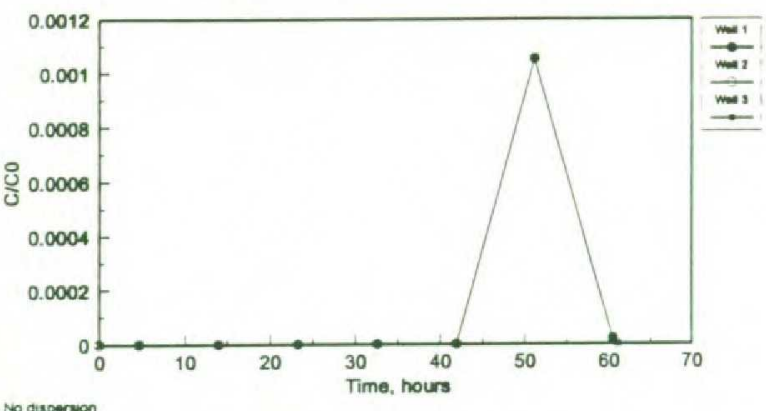

c) Tracer concentration profiles

Figure 6 - A line drive pattern with a higher- $\mathrm{k}$ (100 md) streak

at the other two wells. If we include a lower permeability $(0.01 \mathrm{md})$ zone in the centre of the pattern, as shown in Figure 5, most of the tracer fluid flows around the lower permeability zone towards the production wells. It is clear that the lower permeability region diverts some tracer into well 1 and well 3 , as shown in Figures $5 \mathrm{~b}$ and $5 \mathrm{c}$. If a higher permeability (100 md) streak connects the tracer injection well to production well 1 , as illustrated in Figure 6, all the tracer flows through the high permeability region to well 1 . If the permeability of the streak decreases from $100 \mathrm{md}$ to $2 \mathrm{md}$, as illustrated in Figure 7, most of the tracer would be diverted from well 1 to well 2 . We see that the tracer response depends not only on the permeability but also on the orientation of the heterogeneity pattern. Our model predicts this reasonable behaviour.

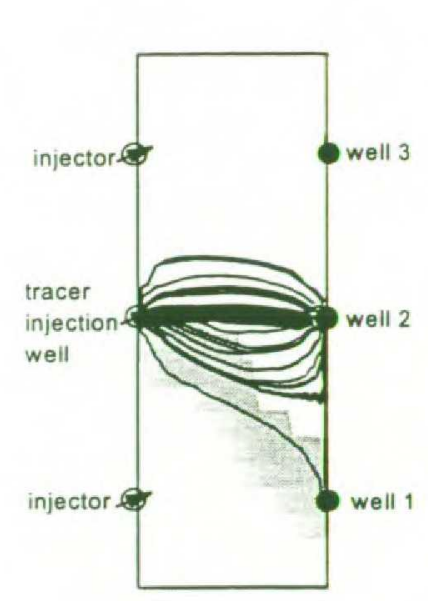

a) Tracer flow lines

Notarosersion

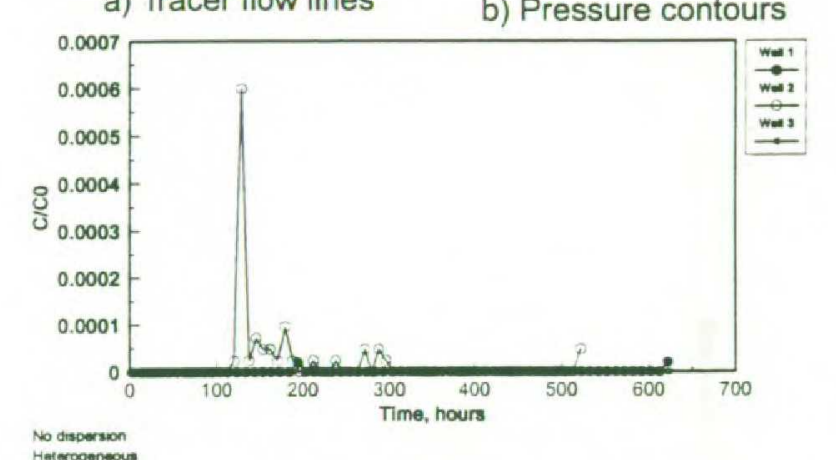

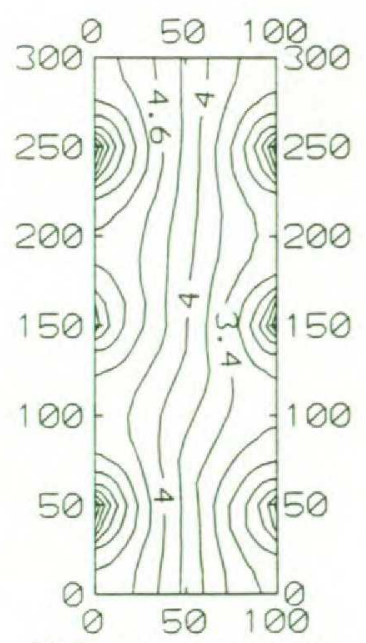

b) Pressure contours

c) Tracer concentration profiles

Figure 7 - A line drive pattern with a higher-k ( 2 md ) streak

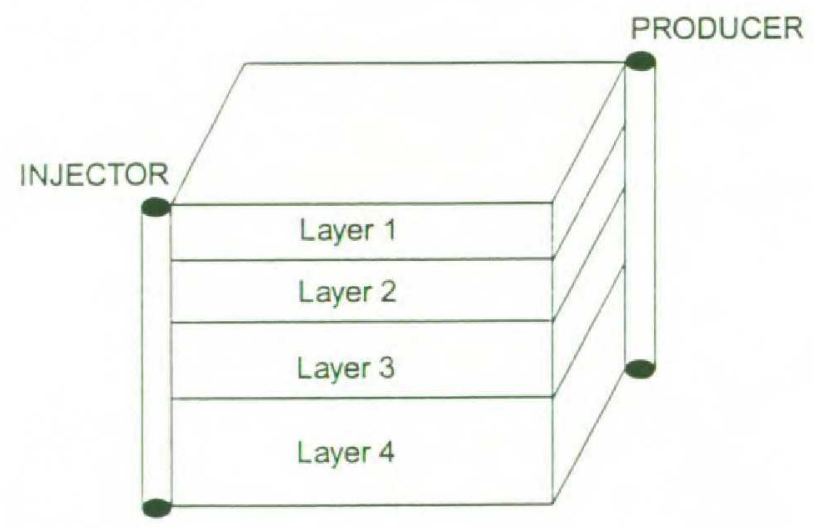

Figure 8 - A multi-layer reservoir ( a quarter five-spot pattern; parameters see Table 1) 
Table 1 - Given parameters of a multi-layer reservoir (see Figure 9)

\begin{tabular}{|c|c|c|c|}
\hline Layer No & $\mathrm{k}(\mathrm{md})$ & $\mathrm{h}(\mathrm{cm})$ & $\mathrm{kh} / \Sigma \mathrm{kh}$ \\
\hline 1 & 119.05 & 38.4 & 0.15 \\
\hline 2 & 83.33 & 73.15 & 0.2 \\
\hline 3 & 90.91 & 83.82 & 0.25 \\
\hline 4 & 100 & 121.92 & 0.4 \\
\hline
\end{tabular}

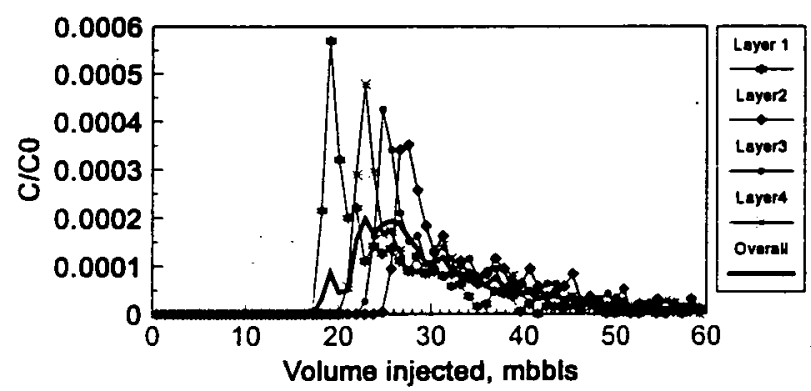

Figure 9 - Tracer concentration profile for the multi-layer reservoir

\subsection{A commingled quarter five-spot pattern}

We can extend the application of our numerical models to consider tracer flow behaviour in a multi-layered porous medium. The model system to illustrate this behaviour is a quarter five spot pattern of a multi-layer reservoir ${ }^{6}$, as shown in Figure 8. It is assumed that there is no communication across layers, and that the injection rate into each layer is linearly proportional to the kh value of each layer. The permeability and the thickness of each layer are listed in Table 1. Tracer concentration profiles are predicted for each layer and for the overall (combined) response from the wellbore, as shown in Figure 9. We see that the concentration response for each layer is quite different. The breakthrough time and the maximum value of the concentration profile for each layer are related strongly to the permeability of each layer. The higher the permeability of a layer, the earlier the breakthrough and the higher the maximum value of the concentration for the layer. The contribution of each layer to the overall tracer response depends on the $\mathrm{kh}$ value of each layer. As illustrated in Figure 9, the first two peaks on the overall response correspond to layer 1 and layer 4 , because of the sharp contrast in their kh values (see Table 1). The contributions from layer 2 and layer 3 are combined forming a flat profile on the overall response curve because of the small difference in their $\mathrm{kh}$ values.

\section{Conclusions}

1. We have shown how our numerical model can be used to predict the tracer flow behaviour, including pressure contours, flow paths and tracer concentration profiles, in heterogeneous reservoirs with different drive patterns.

2. In a quarter five-spot pattern with no-flow boundaries, the presence of a higher-permeability contrast normally reduces the tracer breakthrough time; while a lower-permeability region delays the breakthrough, unless the effective cross-sectional area of the tracer flow is changed dramatically.

3. In a direct line drive pattern with multi-wells, the tracer concentration response at each producer depends on both the value and the distribution of the permeability heterogeneities (bed forms and orientations ).

4. In a commingled multi-layered quarter five-spot pattern, the tracer response from each 
layer, including breakthrough time and the maximum value of tracer concentration, depends on the permeability of each layer; while the overall response depends on the $\mathrm{kh}$ value of each layer. Peaks on the overall response curve corresponding to individual layers depends on the contrast of their kh values.

\section{Symbols}

C - Tracer concentration, fraction;

$\mathrm{C}_{0}$ - Initial tracer concentration, fraction;

h - Formation thickness, cm;

$\mathrm{k}$ - Permeability, md.

\section{Acknowledgement}

The authors wish to thank the Oil \& Gas Division of the UK Department of Trade and Industry for the financial support of this work.

\section{References}

1. Wagner, O.R., The Use of Tracers in Diagnosing Interwell Reservoir Heterogeneities - Field Results, J. Petr. Tech., November, 1977, 1410-1416.

2. Brigham, W.E., and Abbaszadeh-Dehghani, M., Tracer Testing for Reservoir Description, J. Petr. Tech., May, 1987, 519-527.

3. Skjaeveland, S.M. and Kleppe, J., 'Tracer Testing', Recent Advances in Improved Oil Recovery Methods for North Sea Sandstone Reservoir, SPOR Monograph, Chapter 7, 1992, 157-170.

4. Yi, T., Daltaban, T.S. and Dawe, R.A., Prediction of Interwell Tracer Flow Behaviour in Heterogeneous Reservoirs, To appear on Transport in Porous Media, 1995.

5. Yi, T., Daltaban, T.S. and Archer, J.S., Analysis of Interwell Tracer Flow Behaviour in Transient, Two-Phase Heterogeneous Reservoirs, SPE 28901, presented at 94' EUROPEC, London, October, 1994.
6. Abbaszadeh-Dehghani, M. and Brigham, W.E., Analysis of Well-to-Well Tracer Flôtw to Determine Reservoir Heterogeneity, SPE10760, paper presented at the SPE California Regional Meeting, San Francisco, CA, March 24-26, 1982. 